\title{
Concurrent and Local Evaluation of Normal Programs
}

\author{
Rui Marques ${ }^{1}$ and Terrance Swift ${ }^{2}$ \\ 1 CITI, Dep. Informática - FCT, Universidade Nova de Lisboa \\ 2 CENTRIA - Universidade Nova de Lisboa
}

\begin{abstract}
Tabled evaluations can incorporate a number of features, including tabled negation, reduction with respect to the well-founded model, tabled constraints and answer subsumption. Many of these features are most efficiently evaluated using the Local evaluation strategy, which fully evaluates each mutually dependent set of tabled subgoals before returning answers to other subgoals outside of that set. In this paper, we introduce a formalism, Concurrent Local SLG by which multiple threads of computation concurrently perform Local evaluation of the well-founded semantics, and which is a framework for multi-threaded tabling in the XSB system. We prove several properties of Local evaluation within single-threaded tabled computation. We then extend SLG to a model of concurrency and show that the completeness and complexity of SLG are retained when computed by multiple threads. Finally, we extend Local evaluation to concurrent SLG, and show that the properties of Local evaluation continue to hold under concurrency.
\end{abstract}

This paper provides an operational semantics for a type of concurrent TLP that relies on a scheduling strategy called Local evaluation [4]. The model of concurrency adopted is one in which threads of computation execute separate subgoals while sharing completed tables. The main idea behind Local evaluation is that it fully evaluates a single mutually dependent set of tabled subgoals before performing operations (such as returning answers) to subgoals outside of that set. Experiments in several implementations have shown that Local evaluation utilizes space efficiently (see e.g. $[4,10]$ ) and as a result it has been implemented for several Prologs.

Another feature of Local evaluation is shown in an example in [4] in which tabling was used to compute the shortest path between two nodes. When Local evaluation was used the shortest path could be computed in a time proportional to the number of nodes in the graph, while if a non-Local scheduling strategy was used the time was proportional to the number of paths in the graph - i.e. the time was exponential in the number of nodes. Comparing path lengths to compute a shortest path can be considered as an instance of answer subsumption in which answers are retained and propagated only if they are maximal over a partial order or are a monotonic function of answers so far produced.

Using SLG resolution [1] as a basis, this paper presents the following results about concurrent and Local evaluations.

- As analysis of Local evaluation in the literature has been mostly empirical, Local SLG evaluation is formally defined in Section 2 and shown complete 
for queries to normal programs. Properties are derived about dependencies between subgoals in a Local evaluation, about the return of answers, and about the extent of non-completed subgoals in an evaluation.

- Section 3 presents $\mathrm{SLG}_{C}$, an extension of SLG to concurrent evaluations in which completed tables are shared among threads. SLG $\mathrm{S}_{C}$ is complete for queries to normal programs, and its abstract complexity is the same as SLG.

- Concurrent Local SLG (Local $\mathrm{SLG}_{C}$ ) is then defined in Section 3.1. It is shown that properties of Local SLG evaluations extend to the subevaluations performed by each concurrently executing thread, and a property is derived about the structure of dependencies between threads.

- Section 4 sketches the implementation of Local $\mathrm{SLG}_{C}$ in XSB, where the engine design is directly motivated by the preceding results for subgoal and thread dependencies. In addition to having the properties of finite evaluations presented in this paper, XSB's implementation of Local $\mathrm{SLG}_{C}$ has been extended to support tabled constraints, answer subsumption, tabled dynamic code, and space reclamation.

We begin with a review of SLG evaluation.

\section{SLG Evaluation}

This presentation of SLG reformulates the operations of [1] using the model of a forest of trees. However, for reasons of space we make the following restrictions throughout this paper. First, the formal definitions in this paper consider only finite evaluations, although the statements of theorems that are true for transfinite evaluations are not restricted. Second, our definition of Completely Evaluated (Definition 4) does not permit Early Completion. And third, we do not formally define the concept of a supported answer. All of this formalism can be found in the full version of this paper, available at http://www.cs.sunysb.edu/ tswift/papers.html.

Terminology and assumptions We assume the standard terminology of logic programming and an understanding of the well-founded semantics (see [12]). All programs discussed are normal, and defined over a countable language of predicates and function symbols. If $L$ is a literal, the underlying subgoal of $L$ is $L$ if $L$ is positive and $S$ if $L=$ not $S$. A 3-valued interpretation $I$ of a program $P$ is a set of literals defined over the Herbrand base of $P, H_{P}$. For $A \in H_{P}$, if $A \in I, A$ is true in $I$, and if not $A \in I, A$ is false in $I$; otherwise $A$ and not $A$ are undefined in $I$. When $I$ is an interpretation and $A$ is an atom, $\left.I\right|_{A}$ refers to

$\{L \mid L \in I$ and $(L=G$ or $L=\operatorname{not} G)$ and $G$ is in the ground instantiation of $A\}$

The well-founded model of a program $P$ is denoted as $W F M(P)$. In the following sections, we use the terms goal, subgoal, and atom interchangeably. Variant terms are considered to be identical.

The nodes in SLG trees are built from atoms and default literals along with a special type of literal called a delay literal. 
Definition 1 (Delay Literals). A negative delay literal has the form not $A$, where $A$ is a ground atom. A positive delay literal has the form $A_{A n s}^{\text {Subg }}$, where $A$ is an atom whose truth value is based on that of some answer Ans for the subgoal Sub. If $\theta$ is a substitution, then $\left(A_{A n s}^{\text {Subg }}\right) \theta=(A \theta)_{A n s}^{\text {Subg }}$.

The annotations in positive delay literals are used to propagate truth values when a given answer to a given subgoal becomes unconditionally true or false.

Definition 2 ( $S L G$ Trees and Forest). An $S L G$ forest consists of a set of $S L G$ trees. Nodes of $S L G$ trees have the form:

$$
\text { Answer_Template :- DelaySet|GoalList }
$$

or simply fail. In the first form, the Answer_Template is an atom, DelaySet is a set of delay literals and GoalList is a sequence of literals. The second form is called $a$ failure node.

An SLG tree $\mathrm{T}$ is associated with a (possibly empty) marking sequence, which is a sequence of terms possibly preceded by the distinguished term complete. The first element of the marking sequence for $T$ is denoted as marking(T). For a term $t$, setMark $(T, t)$ prepends $t$ to the marking sequence of $T$.

A node $N$ is an answer when it is a leaf node for which GoalList is empty. If the DelaySet of an answer is empty it is termed an unconditional answer, otherwise, it is a conditional answer.

The root node of a given SLG tree has the form $S$ :- $\mid S$ where $S$ is a subgoal - a property ensured by Definition 6 . Thus, within a forest each tree and subgoal are uniquely associated, so when $T$ is an SLG tree in a forest $\mathcal{F}$ whose root node is $S$ :- $\mid S$ it is sometimes convenient to use the terminology $S$ is the root node for $T ; T$ is the tree for $S$; and $S$ is in $\mathcal{F}$. If marking $(T)=$ complete, we refer to both $S$ and $T$ as completed. Until Section 3, marking sequences will either be empty or will contain only the term complete. Literals in a GoalList are resolved by an arbitrary but fixed literal selection strategy. For simplicity, throughout this paper literals are always selected in a left-to-right order.

SLG operations transform one forest of trees into another. One of the operations, Answer RETURn is based on answer resolution, which is extended to take account of delay literals.

Definition 3 (Answer Resolution). Let $N$ be a node $A$ :- $D \mid L_{1}, \ldots, L_{n}$, where $n>0$, and Ans $=A^{\prime}:-D^{\prime} \mid$ an answer whose variables have been standardized apart from $N . N$ is SLG resolvable with Ans if $\exists i, 1 \leq i \leq n$, such that $L_{i}$ and $A^{\prime}$ are unifiable with an mgu $\theta$. The SLG resolvent of $N$ and Ans on $L_{i}$ is:

$$
\left(A:-D \mid L_{1}, \ldots, L_{i-1}, L_{i+1}, \ldots, L_{n}\right) \theta
$$

if $D^{\prime}$ is empty; otherwise the resolvent has the form:

$$
\left(A:-D, L_{i}{ }_{A^{\prime}}^{L_{i}} \mid L_{1}, \ldots, L_{i-1}, L_{i+1}, \ldots, L_{n}\right) \theta
$$

The SLG COMPLETION operation marks a set of trees as complete when they can produce no more useful answers - a condition captured as follows. 
Definition 4 (Completely Evaluated). A set $\mathcal{S}$ of subgoals in a forest $\mathcal{F}$ is completely evaluated if no $S \in \mathcal{S}$ is completed; and if for each $S \in \mathcal{S}$, for each node $N$ in the tree for $S$ :

1. The underlying subgoal of the selected literal of $N$ is completed; or

2. There are no applicable New Subgoal, Program Clause Resolution, Answer Return, Negation Return or Delaying operations (Definition 6) for $N$.

In order to prevent $\mathcal{S}$ from being repeatedly completed, the preceding definition explicitly prohibits $\mathcal{S}$ from containing any completed subgoals.

SLG forests are related to interpretations in the following manner.

Definition 5. Let $\mathcal{F}$ be a forest. The interpretation induced by $\mathcal{F}, I_{\mathcal{F}}$, is the smallest set such that:

- $A$ (ground) atom $A \in I_{\mathcal{F}}$ iff $A$ is in the ground instantiation of some unconditional answer Ans :- $\mid$ in $\mathcal{F}$.

- $A$ (ground) literal not $A \in I_{\mathcal{F}}$ iff $A$ is in the ground instantiation of a completely evaluated subgoal in $\mathcal{F}$, and $A$ is not in the ground instantiation of any answer in $\mathcal{F}$.

An atom $S$ is successful in $\mathcal{F}$ if some tree in $\mathcal{F}$ has an unconditional answer $S . S$ is failed in $\mathcal{F}$ if $S$ is completed and the tree for $S$ contains no answers. An atom $S$ is successful (failed) in $I_{\mathcal{F}}$ if $S^{\prime}$ (not $S^{\prime}$ ) is in $I_{\mathcal{F}}$ for every $S^{\prime}$ in the ground instantiation of $S$. A negative delay literal not $D$ is successful (failed) in a forest $\mathcal{F}$ if $D$ is failed (successful) in $\mathcal{F}$. Similarly, a positive delay literal

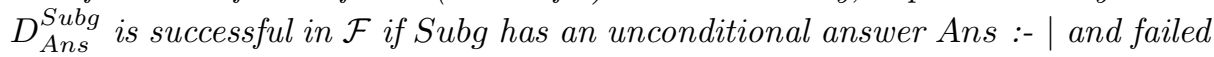
if Subg has no answer with head Ans.

Given these concepts, the SLG operations themselves can be stated.

Definition 6 (SLG Operations). Given a forest $\mathcal{F}_{n}$ of a $S L G$ evaluation of program $P, \mathcal{F}_{n+1}$ may be produced by one of the following operations.

1. New Subgoal: Let $\mathcal{F}_{n}$ contain a non-root node

$$
N=\text { Ans :- DelaySet } \mid G, \text { GoalList }
$$

where $G$ is the selected literal $S$ or not $S$. Assume $\mathcal{F}_{n}$ contains no tree with root subgoal $S$. Then add the tree $S:-\mid S$ to $\mathcal{F}_{n}$.

2. Program Clause Resolution: Let $\mathcal{F}_{n}$ contain a root node $N=S$ :- $\mid S$ and $C$ be a program clause Head :- Body such that Head unifies with $S$ with mgu $\theta$. Assume that in $\mathcal{F}_{n}, N$ does not have a child $N_{\text {child }}=(S:-\mid$ Body $) \theta$. Then add $N_{\text {child }}$ as a child of $N$.

3. ANSWER RETURN: Let $\mathcal{F}_{n}$ contain a non-root node

$$
N=\text { Ans :- DelaySet } \mid \text { S, GoalList }
$$

whose selected literal $S$ is positive. Let Ans be an answer node for $S$ in $\mathcal{F}_{n}$ and $N_{\text {child }}$ be the $S L G$ resolvent of $N$ and Ans on $S$. Assume that in $\mathcal{F}_{n}, N$ does not have a child $N_{\text {child. }}$ Then add $N_{\text {child }}$ as a child of $N$. 
4. Negation Return: Let $\mathcal{F}_{n}$ contain a leaf node

$$
N=\text { Ans :- DelaySet|not S, GoalList. }
$$

whose selected literal not $S$ is ground.

(a) Negation Success: If $S$ is failed in $\mathcal{F}_{n}$, then create a child for $N$ of the form: Ans :- DelaySet|GoalList.

(b) Negation Failure: If $S$ succeeds in $\mathcal{F}_{n}$, then create a child for $N$ of the form fail.

5. Delaying: Let $\mathcal{F}_{n}$ contain a leaf node $N=$ Ans :- DelaySet|not S, GoalList, such that the selected literal npt $S$ is ground, $S$ is in $\mathcal{F}_{n}$, but $S$ is neither successful nor failed in $\mathcal{F}_{n}$. Then create a child for $N$ of the form Ans :- DelaySet, not S|GoalList.

6. Simplification: Let $\mathcal{F}_{n}$ contain a leaf node $N=$ Ans :- DelaySet|, and let $L \in$ DelaySet

(a) If $L$ is failed in $\mathcal{F}$ then create a child fail for $N$.

(b) If $L$ is successful in $\mathcal{F}$, then create a child Ans :- DelaySet' for $N$, where DelaySet ${ }^{\prime}=$ DelaySet $-L$.

7. Completion: Given a completely evaluated set $\mathcal{S}$ of subgoals (Definition 4), for each $S \in \mathcal{S}$, setMark $(T$, complete), where $T$ is the tree for $S$.

8. Answer Completion: Given a set of unsupported answers $\mathcal{U} \mathcal{A}$, create a failure node as a child for each answer Ans $\in \mathcal{U} \mathcal{A}$.

In the above definition, the ANSWER COMPLETION operation relies on the concept of unsupported answers. Unsupported answers are conditional answers that are false in the well-founded model, and reflect certain unfounded sets in that model. While necessary for completeness of SLG, the Answer CompleTION operation is not affected by local or concurrent evaluations, so for reasons of space, we omit its formal definition (see the full version of this paper).

SLG Evaluations An SLG evaluation consists of a (possibly transfinite) sequence of forests. However as noted, we restrict definitions of evaluations to be finite for reasons of space (see the full version of this paper for the general case).

Definition 7 (SLG Evaluation). Given a program $P$ and goal $G$, an $S L G$ evaluation $\mathcal{E}$ is a sequence of $S L G$ forests $\mathcal{F}_{0}, \mathcal{F}_{1}, \ldots, \mathcal{F}_{\beta}$, such that:

- $\mathcal{F}_{0}$ is the forest containing a single tree $\mathrm{G}$ :- $\mid \mathrm{G}$

- For each successor ordinal, $n+1 \leq \beta, \mathcal{F}_{n+1}$ is obtained from $\mathcal{F}_{n}$ by an application of an SLG operation from Definition 6.

If no operation is applicable to $\mathcal{F}_{\beta}, \mathcal{F}_{\beta}$ is called a final forest of $\mathcal{E}$. If $\mathcal{F}_{\beta}$ contains a leaf node with a non-ground selected negative literal, it is floundered.

The correctness is formulated as follows for transfinite evaluations

Theorem 1 ([1]). Let $\mathcal{E}$ be an $S L G$ evaluation of a goal to a program $P$. Then $\mathcal{E}$ has a final forest $\mathcal{F}$. Let $A$ be an atom such that $A:-\mid A$ is the root of some tree in $\mathcal{F}$. Then if $\mathcal{F}$ is non-floundered, $\left.W F M(P)\right|_{A}=\left.I_{\mathcal{F}}\right|_{A}$. 


\section{Local SLG Evaluations}

As noted above, a Local SLG evaluation fully evaluates each mutually dependent set of tabled subgoals before performing operations to subgoals outside of that set. We begin to formalize that notion by defining what it means for one subgoal to depend on another.

Definition 8 (Subgoal Dependency Graph). Let $\mathcal{F}$ be a forest, and let $S_{1}$ :- $\mid S_{1}$ be the root of a non-completed tree in $\mathcal{F}$. The subgoal $S_{1}$ directly depends on a subgoal $S_{2}$ iff $S_{2}$ is not completed in $\mathcal{F}$, and there is some node $N$ in the tree for $S_{1}$ such that $S_{2}$ is the underlying subgoal of the selected literal of $N$.

The Subgoal Dependency Graph of $\mathcal{F}, \operatorname{SDG}(\mathcal{F})=(\mathrm{V}, \mathrm{E})$, is a directed graph in which $\left(S_{i}, S_{j}\right) \in E$ iff subgoal $S_{i}$ directly depends on subgoal $S_{j}$, and $V$ is the underlying set of $E$. $S_{1}$ "depends on" $S_{2}$ in $\mathcal{F}$ is there is a path from $S_{1}$ to $S_{2}$ in $\operatorname{SDG}(\mathcal{F})$.

Since the SDG of a forest is a directed graph, it can be partitioned into disjoint sets of strongly connected components, or SCCs, where a node with no outgoing edges is considered to be in a trivial SCC. We refer to a given SCC by the set of its vertices (subgoals), and distinguish independent SCCs.

Definition 9 (Independent SCC). A strongly connected component $\mathcal{S}$ is independent if $\forall S \in \mathcal{S}$ : if $S$ depends on some $S^{\prime}$, then $S^{\prime} \in \mathcal{S}$.

By Definition 9 it is straightforward that a trivial SCC is independent, and that each independent component is maximal - i.e. contained in no larger SCC. Local evaluation, then, performs operations on independent SCCs. Formally:

Definition 10 (Local SLG Evaluation). Given a program $P$ and goal $G$, $a$ Local $S L G$ evaluation $\mathcal{E}$ is a sequence of $S L G$ forests $\mathcal{F}_{0}, \mathcal{F}_{1}, \ldots, \mathcal{F}_{\beta}$, such that:

1. $\mathcal{F}_{0}$ is the forest containing a single tree $\mathrm{G}$ :- $\mid \mathrm{G}$

2. For each successor ordinal, $n+1 \leq \beta, \mathcal{F}_{n+1}$ is obtained from $\mathcal{F}_{n}$ by an application of an $S L G$ operation from Definition 6 such that:

(a) if a NEw SubGoAl operation is applied to create a tree $S:-\mid S$ then $S$ is the underlying subgoal of a selected literal in a tree whose root subgoal is in an independent $S C C$ of $S D G\left(\mathcal{F}_{n}\right)$;

(b) a Program Clause Resolution, Answer Return, Negation ReTURN or DELAYING operation is only applied to a node on a tree whose root subgoal is in an independent $S C C$ of $S D G\left(\mathcal{F}_{n}\right)$;

$\mathcal{E}$ is delay avoiding if no DELAYING operation is performed in a forest if any other operation is applicable.

In the transfinite extension of Definition 10, a Local (SLG) evaluation works as an unrestricted SLG evaluation whenever an independent SCC does not exist in a forest, leading to the following theorem.

Theorem 2 (Completeness of Local Evaluation). Let $P$ be a program and $G$ a goal. Then there exists an $S L G$ evaluation $\mathcal{E}$ of $G$ against $P$ with final forest $\mathcal{F}$ if and only if there exists a local $S L G$ evaluation $\mathcal{E}^{L}$ of $G$ against $P$ with final forest $\mathcal{F}^{L}$ such that $\left.I_{\mathcal{F}}\right|_{G}=\left.I_{\mathcal{F}^{L}}\right|_{G}$. 
While Local evaluation is ideally complete for the well-founded semantics, its importance arises from its efficiency for certain classes of programs, along with properties that can be used to ensure the correctness of implementations. The first such property is:

Theorem 3. Let $\mathcal{E}^{L}$ be a finite Local $S L G$ evaluation. For each $\mathcal{F}$ in $\mathcal{E}^{L}$ $S D G(\mathcal{F})$ has one and only one independent $S C C$.

Theorem 3 implies the following corollary which will be used by the implementation described in Section 4.

Corollary 1. Let $\mathcal{E}^{L}$ be a finite Local $S L G$ evaluation. For each $\mathcal{F}$ in $\mathcal{E}^{L}$ there is at most one incoming edge for each maximal $S C C$ in $S D G(\mathcal{F})$.

The following corollary captures the notion that in a Local evaluation, a subgoal may only return answers out of its SCC once its SCC has been completed.

Corollary 2. In any forest $\mathcal{F}$ of a Local $S L G$ evaluation, if an answer $A$ is used in an ANSWER RETURN operation to a node in a tree with root subgoal $S$, then the tree for $A$ has been completed, or is in the same $S C C$ as $S$ in $S D G(\mathcal{F})$.

Corollary 2 has practical importance for answer subsumption since it implies that no answer $A$ need be returned out of an SCC if the model entails an answer that is preferred to $A$ - only the preferred answer need be returned. In addition, it is easy to see that if Local evaluation were extended to ensure that all appropriate SimplifiCATION and ANSwER COMPLETION operations are performed for an independent SCC just after it has been completed, the following statement also holds. If a forest in Local evaluation contains a conditional answer $A=S$ :- $D \mid$ and $S$ is successful or failed in $\mathcal{F}, A$ will never be propagated outside of the SCC. This strategy reduces the overall number of SimpLIFICATION and Answer Completion operations and has been adopted by the XSB engine when computing non-stratified programs. The space efficiency of Local evaluation is stated as follows:

Theorem 4. Let $\mathcal{E}^{L}$ be a finite delay-avoiding Local evaluation of a goal $G$ to a program $P$, and let $\mathcal{E}$ be an $S L G$ evaluation of $G$ to a $P$. Then for any forest $\mathcal{F}^{L}$ in $\mathcal{E}^{L}$, there exists a forest $\mathcal{F}$ in $\mathcal{E}$ such that $S D G\left(\mathcal{F}^{L}\right)$ is a subgraph of $S D G(\mathcal{F})$.

\section{Sharing Completed Tables in a Concurrent Evaluation}

Rather than starting with a single top-level atomic query, a concurrent SLG, $\mathrm{SLG}_{C}$, evaluation is initialized with a set of atomic queries, such that each atomic query is evaluated by a different thread of computation. In this model of concurrency, threads share only completed tables so that a thread is prevented from consuming answers from a (non-completed) table owned by another thread. This disallows consume-producer models of concurrency and implies that different threads may not collaborate to evaluate subgoals within a single SCC. 
However as discussed below, within a Local evaluation the restriction may not be binding since Local evaluations prevent consumer-producer models by their nature, and since the scope of an SCC in a Local evaluation is relatively small.

Formally, this model of concurrency marks every non-completed tree in a given forest with a thread identifier (cf. Definition 2). As terminology, if $N$ is a node in a tree $T$, marking $(N)$ denotes marking $(T)$, and if $S$ is a subgoal marking $(S)$ denotes marking $(T)$, where $T$ is the tree for $S$.

Definition 11 (Thread). A thread identifier is an element of a set of terms that does not include the term complete. Given an $S L G$ forest $\mathcal{F}$ in an evaluation $\mathcal{E}$, a thread state is the maximal set $\mathcal{T}$ of trees in $\mathcal{F}$ such that for all $T \in \mathcal{T}$ marking $(T)=t$ where $t$ is a thread identifier. $A$ thread in $\mathcal{E}$ is the sequence of thread states for a given marking. A thread is active in $\mathcal{F}$ if its thread state in $\mathcal{F}$ is non-empty.

Let $S$ be a subgoal, $T$ the tree for $S$, and $N$ a node in a forest. $N$ is thread compatible with $S$ if marking $(T)=$ complete or marking $(T)=\operatorname{marking}(N)$.

SLG $_{C}$ uses SLG forests and other notions from Definitions 1-5, but differs in that certain $\mathrm{SLG}_{C}$ operations may create or change thread markings, and markings may restrict the applicability of operations based on whether a node and subgoal are thread compatible according to the previous definition. Definition 12 presents a new operation called UsURPATION, along with those operations that differ from Definition 6 where the difference in each altered operation is underlined.

Definition 12 (SLG $\mathbf{S L}_{C}$ Operations). Given an $S L G$ forest $\mathcal{F}_{n}, \mathcal{F}_{n+1}$ may be produced by one of the following operations.

1. New Subgoal: Let $\mathcal{F}_{n}$ contain a non-root node

$$
N=\text { Ans :- DelaySet } \mid G, \text { GoalList }
$$

where $G$ is the selected literal $S$ or not $S$. Assume $\mathcal{F}_{n}$ contains no tree with root subgoal $S$. Then add the tree $T=S$ :- $\mid S$ to $\mathcal{F}_{n}$, and setMark $(T$, marking $(N))$.

2. ANSWER RETURN: Let $\mathcal{F}_{n}$ contain a non-root node

$$
N=\text { Ans :- DelaySet } \mid \text { S, GoalList }
$$

whose selected literal $S$ is positive. Let Ans be an answer node for $S$ in $\mathcal{F}_{n}$ such that $N$ is thread compatible with $S$ and let $N_{\text {child }}$ be the SLG resolvent of $N$ and Ans on $S$. Assume that in $\mathcal{F}_{n}, N$ does not have a child $N_{\text {child }}$. Then add $N_{\text {child }}$ as a child of $N$.

3. Negation Return: Let $\mathcal{F}_{n}$ contain a leaf node

$$
N=\text { Ans :- DelaySet|not S, GoalList. }
$$

whose selected literal not $S$ is ground where $N$ is thread compatible with $S$.

(a) Negation Success: If $S$ is failed in $\mathcal{F}_{n}$, then create a child for $N$ of the form: Ans :- DelaySet|GoalList.

(b) Negation Failure: If $S$ succeeds in $\mathcal{F}_{n}$, then create a child for $N$ of the form fail. 
4. Completion: Given a completely evaluated set $\mathcal{S}$ of subgoals such that for all $S \in \mathcal{S}$, marking $(S)=t$, then for each $S \in$

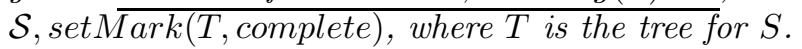

5. UsurPation: Let $\mathcal{S}$ be a set of subgoals in deadlock (Definition 13), $S_{U} \in \mathcal{S}$, and $T_{U}$ the tree for $S_{U}$. For each $S \in \mathcal{S}$, setMark $\left(T, \operatorname{marking}\left(T_{U}\right)\right)$.

The thread compatibility restrictions can mean that an SLG operation is applicable in a given forest, but that the corresponding $\mathrm{SLG}_{C}$ operation is not. The UsurPATION operation is designed to address cases where SLG $\mathrm{G}_{C}$ operations might get stuck - which are formalized as situations of deadlock.

Definition 13 (Deadlock). A set $\mathcal{S}$ of subgoals in a forest $\mathcal{F}$ is in deadlock if:

1. For each $S \in \mathcal{S}$ there are no applicable New Subgoal, Program Clause Resolution, Answer Return, Negation Return or Delaying operations of Definition 12; and

2. There exists no $\mathcal{S}^{\prime}$ such that $\mathcal{S} \subseteq \mathcal{S}^{\prime}$ and $\mathcal{S}^{\prime}$ is completely evaluated in $\mathcal{F}$.

Example 1. As defined, $\mathrm{SLG}_{C}$ evaluations may use any scheduling strategy, and are not restricted to Local evaluations. They also begin with a set of goals rather than with a single goal. Figure 1 illustrates a simple, non-Local, $\mathrm{SLG}_{C}$ evaluation of the goal $\{\mathrm{a}(\mathrm{X}), \mathrm{b}(\mathrm{X})\}$ to the program $P_{2}$, where $\mathrm{a}(\mathrm{X})$ is initially marked with thread identifier 1 and $b(X)$ with thread identifier 2. Through NEw SUBGOAL operations, trees for $\mathrm{c}(\mathrm{X})$ and $\mathrm{e}(\mathrm{X})$ are created and associated with thread identifier 1 , while $d(X)$ is created and associated with thread identifier 2. Evaluation continues until there is a deadlock, as shown in Figure 1b. Note in Figure 1b, that while there is an answer that could be returned to the node $e(1):-\mid d(X)$ in a non-Local evaluation, the node is associated with thread identifier 1 , while the answer is associated with thread identifier 2 so that the return is prohibited by the thread compatibility restrictions. UsURPATION is the only operation applicable to this forest; assume that thread identifier 1 performs the Usurpation, marking trees for $\mathrm{c}(\mathrm{X}), \mathrm{d}(\mathrm{X})$, and $\mathrm{e}(\mathrm{X})$ with identifier 1 . Afterward, an answer for e(1) is derived, leading to Figure 1c. Further Answer RETURN operations lead to Figure 1d.'v All of the subgals in thread identifier 1 have been completely evaluated, but the subgoal $b(X)$ in thread identifier 2 cannot be completely evaluated until the answer for $d(X)$ is resolved with the node $b(X):-I d(X)$. Since a completed subgoal is thread compatible with any thread, once $d(X)$ is completed, the answer for $d(X)$ can be resolved.

The definition of a $\mathrm{SLG}_{C}$ evaluation is nearly the same as for SLG (Definition 7), but is initialized so that each atomic query in the set of goals it is presented with is marked with a different thread identifier (Its formal, transfinite, definition can be found in the full version of this paper). In addition, SLG $_{C}$ forests are based on Definition 2, so the definition of an interpretation induced by a forest is identical in both frameworks, leading to the following theorem.

Theorem 5 (Correctness of $\mathbf{S L G}_{C}$ ). Let $P$ be a program and $\mathcal{G}$ a finite nonempty set of goals. Then a $S L G_{C}$ evaluation of $\mathcal{G}$ against $P$ exists with final state 


$$
\begin{array}{ll}
a(X):-c(X) \cdot b(X):-d(X) \cdot c(X):-e(1), d(X) \cdot \begin{array}{l}
d(X):-c(X) . \\
d(1) .
\end{array} & \\
e(1):-d(X) &
\end{array}
$$

\begin{tabular}{|c|c|c|c|c|}
\hline $\mathrm{a}(\mathrm{X}):-\mid \mathrm{a}(\mathrm{X})[1]$ & $\mathrm{b}(\mathrm{X}):-\mid \mathrm{b}(\mathrm{X})[2]$ & $c(X):-\mid c(X)[1]$ & $\mathrm{d}(\mathrm{X}):-\mid \mathrm{d}(\mathrm{X})[2]$ & $\mathrm{e}(1):-\mid \mathrm{e}(1)[1]$ \\
\hline$a(X):-\mid c(X)$ & $\mathrm{b}(\mathrm{X}):-\mid \mathrm{d}(\mathrm{X})$ & $\mathrm{c}(\mathrm{X}):-\mid \mathrm{e}(1), \mathrm{d}(\mathrm{X})$ & $\mathrm{d}(\mathrm{X}):-\mid \mathrm{c}(\mathrm{X})$ & $\mathrm{e}(1):-\mid \mathrm{d}(X)$ \\
\hline
\end{tabular}

(a) The Program $P_{2}$

\begin{tabular}{|c|c|c|c|c|}
\hline $\begin{array}{l}a(X):-\mid a(X)[1] \\
a(X):-\mid c(X)\end{array}$ & $\begin{array}{l}\mathrm{b}(\mathrm{X}):-\mid \mathrm{b}(\mathrm{X})[2] \\
\mathrm{b}(\mathrm{X}):-\mid \mathrm{d}(\mathrm{X})\end{array}$ & $\begin{array}{l}c(X):-\mid c(X)[1] \\
c(X):-\mid e(1), d(X) .\end{array}$ & $d(X):-|c(X) \quad d(1):-|$ & $\begin{array}{l}\mathrm{e}(1):-\mid \mathrm{e}(1)[1] \\
\mathrm{e}(1):-\mid \mathrm{d}(\mathrm{X}) \\
\mathrm{e}(1):-\mid\end{array}$ \\
\hline
\end{tabular}

(b) State $\alpha$ : Deadlock

(c) State $\beta$ : Answer for e(1)

$\underset{\mathrm{a}(1):-\mid}{\mathrm{a}(X):-\mid \mathrm{a}(\mathrm{X})[1]} \mathrm{b(X):-| \textrm {b } ( X ) [ 2 ]}$

(d) State $\gamma$ : Complete Evaluation for Thread Identifier 1

Fig. 1. A non-Local SLG ${ }_{C}$ Evaluation of $P_{2}$

$\widehat{\mathcal{F}}$, iff for every $G_{i} \in \mathcal{G}$ there exists an $S L G$ evaluation of $G_{i}$ against $P$ with final state $\mathcal{F}^{i}$ and $I_{\widehat{\mathcal{F}}}=\left(\bigcup I_{\mathcal{F}^{i}}\right)$.

The completeness portion of the theorem follows from a demonstration that for any SLG operation on a forest, an equivalent SLG S $_{C}$ operation is applicable after zero or more USURPATION operations. The following theorem bounds the number of USURPATION operations in a finite evaluation, which implies that the abstract complexity of $\mathrm{SLG}_{C}$ is the same as that of SLG.

Theorem 6 (Complexity of Usurpation). Let $\mathcal{E}$ be a finite $S L G_{C}$ evaluation with final forest $\mathcal{F}$, and $S_{\mathcal{F}}$ the set of all subgoals in $\mathcal{F}$. Then there are at most $\left|\mathcal{S}_{\mathcal{F}}\right|$ UsuRPATION operations performed.

\subsection{Concurrent Local Evaluations}

In $\mathrm{SLG}_{C}$ the Subgoal Dependency Graph (Definition 8) can be partitioned into disjoint sub-graphs for each thread state of a forest. 
Definition 14 (Thread Subgoal Dependency Graph). For each thread state $t$ in a forest $\mathcal{F}$, the Thread Subgoal Dependency Graph of $t$ $($ Thread_SDG $(\mathcal{F}, t)$ ) consists of the sub-graph of $S D G(\mathcal{F})$ determined by subgoals in $\mathcal{F}$ whose marking is $t$.

Local $\mathrm{SLG}_{C}$ evaluation is based on independent SCCs within Thread SDGs, rather than within a global SDG.

Definition 15 (Local $\mathbf{S L G}_{C}$ ). Given a program $P$, a set $\mathcal{T}$ of thread identifiers, and a finite non-empty set $G$ of goals, a Local $S L G_{C}$ evaluation $\mathcal{E}$ is a sequence of forests $\mathcal{F}_{0}, \mathcal{F}_{1}, \ldots, \mathcal{F}_{\beta}$, such that:

1. $\mathcal{F}_{0}$ is a set-minimal forest containing the trees $T_{i}=G_{i}$ :- $\mid G_{i}$, for each $G_{i} \in$ $\mathcal{G}$, where for each $T_{i}$ there is a $t_{i} \in \mathcal{T}$ such that marking $\left(T_{i}\right)=t_{i}$, and $t_{i} \neq t_{j}$ if $i \neq j$.

2. For each successor ordinal, $n+1 \leq \beta, \mathcal{F}_{n+1}$ is obtained from $\mathcal{F}_{n}$ by an application of an operation from Definition 12 such that:

(a) if a New Subgonl is applied to create a tree $T=S$ :- $\mid S$ then $S$ is the underlying subgoal of a selected literal in a tree whose root subgoal is in an independent $S C C$ of Thread_SDG $\left(\mathcal{F}_{n}\right.$, marking $\left.(T)\right)$;

(b) a Program Clause Resolution, Answer Return, NegaTION RETURN or DELAYING operation is only applied to a node on a tree whose root subgoal is in an independent SCC of Thread_SDG $\left(\mathcal{F}_{n}\right.$, marking $\left.(T)\right)$.

This finitary definition can be extended to the transfinite evaluations, leading to the following theorem.

Theorem 7 (Correctness of Local $\mathbf{S L G}_{C}$ ). Let $P$ be a program and $\mathcal{G}$ a finite non-empty set of goals. Then a Local $S L G_{C}$ evaluation of $\mathcal{G}$ against $P$ exists with final state $\widehat{\mathcal{F}}$, iff every $G_{i} \in \mathcal{G}$ there exists an $S L G$ evaluation of $G_{i}$ against $P$ with final state $\mathcal{F}^{i}$ and $I_{\widehat{\mathcal{F}}}=\left(\bigcup I_{\mathcal{F}^{i}}\right)$.

The following theorem is an analogue of Theorem 3, and implies that each

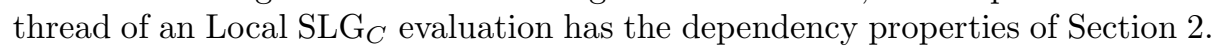

Theorem 8. Let $\mathcal{F}$ be a forest in a finite Local $S L G_{C}$ evaluation. Then for each active thread $t$ in $\mathcal{F}$, Thread_SDG $\mathcal{F}, t)$ has one and only one independent $S C C$.

The Thread Dependency Graph can be seen as a homomorphism of the SDG of a given $\mathrm{SLG}_{C}$ forest.

Definition 16 (Thread Dependency Graph). Let $t_{1}$ and $t_{2}$ be two active threads in a $S L G$ forest $\mathcal{F}$. $t_{1}$ directly depends on $t_{2}$ if there exist a subgoal in $t_{1}$ that directly depends on a subgoal in $t_{2}$ (according to Definition 8). The Thread Dependency Graph $\operatorname{TDG}(\mathcal{F})=(\mathrm{V}, \mathrm{E})$ of $\mathcal{F}$ is a directed graph where $V$ is the set of active threads in $\mathcal{F}$ and $\left(t_{i}, t_{j}\right) \in E$ iff $t_{i}$ directly depends on $t_{j}$.

Based on the thread dependency graph, the following theorem shows that any thread depends on at most one other thread. 
Theorem 9. Let $\mathcal{F}$ be a forest in a finite Local $S L G_{C}$ evaluation. Then for each node in $T D G(\mathcal{F})$ there is at most one outgoing edge.

As a practical matter, this theorem indicates that each thread of computation will wait on the results from at most one other thread. so that the thread communication and dependency detection required to implement the USURPATION operation will be relatively simple.

\section{Implementing $\mathrm{SLG}_{C}$ in the SLG-WAM}

We summarize the changes made to XSB's SLG-WAM in order to implement Local $\mathrm{SLG}_{C}$. Our discussion omits numerous optimizations required for efficiency. In particular, due to space restrictions we do not discuss the propagation of subgoal dependencies between threads, or the handling of subgoals that have been usurped multiple times (see [8] for details). We first describe Local $\mathrm{SLG}_{C}$ for definite programs before considering negation.

Since XSB's SLG-WAM implements Local evaluation, it is evident from Section 3 that the main addition is the USURPATION operation, which mainly affects the SLG-WAM tabletry instruction. This instruction occurs at the entry point of a tabled predicate when a tabled subgoal Subg is called. In the sequential

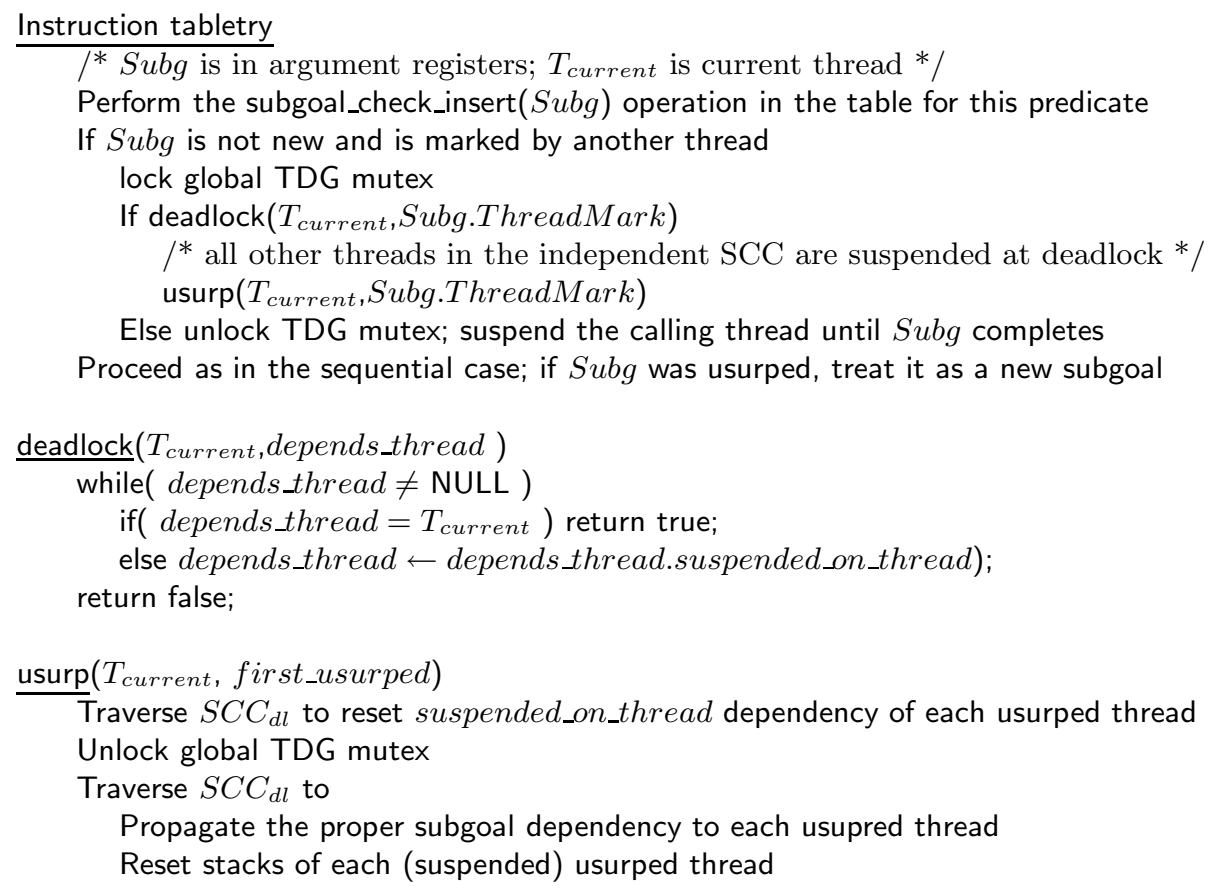

Fig. 2. Summary of Concurrent Local SLG implementation in the SLG-WAM. 
SLG-WAM tabletry is essentially responsible for determining whether a NEW SubGOAL operation is required. The instruction first determines whether $S u b g$ is in the table using its representation in the WAM argument registers. If $S u b g$ is not in the table, a NEW SUBGOAL operation is effectively performed. Subg will have been copied to the table during the check; and a generator choice point is created to backtrack through program clauses, to check whether the subgoal's SCC has been fully evaluated, and to schedule Answer RETURN operations if the SCC is not fully evaluated. On the other hand, if Subg is in the table, tabletry creates a consumer choice point to backtrack through any answers to Subg in the table and thereby perform ANSWER RETURN operations.

Extensions to tabletry for Local $\mathrm{SLG}_{C}$ are summarized in Figure 2. If $S u b g$ is new, it is copied into the table as in the sequential case, but in order to represent the TDG a thread identifier is associated with Subg. For this association the subgoal frame, a structure containing information about each tabled subgoal, is extended with a ThreadMark cell. The essential difference from the sequential case of tabletry occurs when $S u b g$ is not new and is currently marked by another thread (and therefore not marked as completed). In this case deadlock detection is performed: if a deadlock is not found, the calling thread $T_{\text {current }}$ suspends as it does not have any applicable Local SLG ${ }_{C}$ operations; otherwise $T_{\text {current }}$ performs a UsURPATION operation. In addition to changes in tabletry, a change is made to the SLG-WAM completion instruction so that any thread suspended on $S u b g$ is awakened when Subg is completed (a condition variable based on the predicate symbol of $S u b g$ is used for this awakening).

The design of deadlock detection in the SLG-WAM relies on Theorem 9, which states that each thread may be suspended on at most one other thread. The SLG-WAM adds a suspended_on_thread field to the context of each thread to denote any thread dependency. As shown in Figure 2, when a thread $T_{\text {current }}$ performs deadlock detection, it starts by checking whether the thread marking Subg is suspended using this suspended_on_thread field: if the thread is not suspended, $T_{\text {current }}$ may suspend without fear of deadlock and it will be awakened when $S u b g$ is completed. If the marker of $S u b g$ is suspended, the deadlock detection code follows the suspended_on_thread field. By Theorem 9, any loop in the TDG must be a simple cycle so that deadlock detection is a simple while loop that exits in one of two cases. If $T_{\text {current }}$ is found in the suspended_on_thread field for one of the traversed threads, then $T_{\text {current }}$ depends (transitively) on itself and deadlock occurs; otherwise if the suspended_on_thread field of a traversed thread is null, $T_{\text {current }}$ transitively depends on a subgoal that is actively being computed.

The fact that the thread dependencies for deadlocked threads form a simple cycle also underlies the control flow of the usurp() function which consists of two traversals of the deadlocked $T D G$ cycle, denoted $S C C_{d l}$. Each traversal begins with the thread that marks Subg. In the first traversal, the usurping thread $T_{\text {current }}$ updates the TDG, setting the suspended_on_thread field of each usurped thread to its own id. Adjusting the TDG must be performed under global mutual exclusion: otherwise two concurrently usurping threads might produce an incoherent $T D G$. In the second traversal, which is not under mutual exclusion, 
the execution stacks in each usurped thread $T_{\text {usurped }}$ are examined and manipulated - an operation that is safe since $T_{\text {usurped }}$ has suspended on a subgoal due to thread compatibility restrictions. The manipulation ensures that $T_{\text {usurped }}$ will no longer generate answers for usurped subgoals that it has marked, but rather will be set to consume answers. This stack manipulation is considerably simplified by the property that the $S D G$ for $T_{\text {usurped }}$ will depend on a single usurped subgoal $S_{T_{\text {usurped }}}$ - the first subgoal in $S C C_{d l}$ that $T_{\text {usurped }}$ encountered. (the property is implied by Corollary 1 together with Theorem 8). However to determine $S_{T_{u s u r p e d}}$, both subgoal dependencies contained in $T_{\text {usurped }}$ and subgoal dependencies across usurped threads must be considered. Accordingly, usurp() also propagates cross-thread dependencies (the actual mechanism is not shown in this summary) and uses these dependencies when resetting the stacks of $T_{\text {usurped }}$. As a result, when $T_{\text {usurped }}$ is awakened it will call $S_{T_{\text {usurped }}}$ again from scratch and backtrack through the answers of the completed subgoal.

This approach has the virtue of conceptual simplicity, but any partial computations for the usurped subgoals are lost, and will be recomputed by the usurping thread. Theorem 6 states that the maximal number of UsURPATION operations in a $\mathrm{SLG}_{C}$ evaluation is linear in $\operatorname{atoms}(P)$, the number of atoms in a program $P$. In $[8]$ it is shown that UsURPATION operations affect only constanttime operations so that even if answers for usurped subgoals are recomputed, the complexity of the well-founded semantics is unaffected.

Extensions for Negation and Answer Subsumption As suggested by the changed operations in Definition 12, the SLG-WAM requires few modifications beyond those presented to extend Local SLG $\mathrm{S}_{C}$ to the well-founded semantics. Consider first stratified programs. In the SLG-WAM, if the underlying (tabled) subgoal Subg of a selected negative literal is not new and not complete, the computation path "suspends" and resumes only when Subg has been completed. These operations are essentially the same as the interactions between threads so far described. In the case of non-stratified negation the first new operation to consider is the Delaying operation. If $S u b g$ is involved in a loop through negation, the resumption mechanism is the same except that a bit in the subgoal frame of $S u b g$ is set to indicate that $S u b g$ was delayed rather than completed. Several cycles of delaying may be needed before $S u b g$ is finally completed, but each cycle may be handled by the thread suspension and usurpation mechanisms described. When Subg is completed, any Simplification operations for its SCC are performed before awakening any threads suspended on $S u b g$, so that Simplification is not affected by the concurrency mechanisms. Beyond negation, answer subsumption is implemented as an extension to the SLG-WAM new_answer operation which is unaffected by Local SLG ${ }_{C}$.

Performance Several performance studies have been made on tabling with Local $\mathrm{SLG}_{C}[6,8,7]$. We focus on tests of scalability in which a list of $M$ queries is distributed to $N$ threads and the elapsed time measured. [7] measured the use of Local $\mathrm{SLG}_{C}$ on programs which analyzed configuration reachability for various extensions of Petri nets. Depending on particular formalism for the net, the programs were definite, or used well-founded negation, tabled constraints or answer subsumption. For nearly all of these benchmarks, left-recursive reacha- 
bility of the form reachable(bound, free)) scaled perfectly to 4 processors (the number available for this experiment).

In constrast, [8] measured scalability on a worst case: where multiple threads concurrently evaluated right recursion on random graphs of varying densities, using queries of the form rightRec (bound, free). Observe that for right-recursion over a graph, the connectivity of the $S D G$ directly reflects the connectivity of the graph. Consider properties of a random graph of $V$ vertices (cf. [11]). If each vertex has at most 1 edge there can be no cycles; if each vertex has between 1 and $\ln (V)$ edges the graph (and $S D G$ ) is likely to be split up into several SCCs; while if each vertex has $\ln (V)$ or more edges the graph is likely to be connected, with the $S D G$ consisting of a single SCC. While somewhat preliminary, [8] indicates that the number of deadlocks are relatively small. For graphs with between 1 and $\ln (V)$ edges per vertex, this is either because the graphs do not contain large SCCs or because the subgoals in these SCCs are quickly completed. For fully connected graphs, each thread is usurped at most once. As expected, scalability is poor for the connected graphs as usurped threads must wait for the SCC to be evaluated. However, the elapsed time for the Local $\mathrm{SLG}_{C}$ is never worse than that for a Local single-threaded evaluation on any graph. In other words, for these benchmarks the implementation of Local $\mathrm{SLG}_{C}$ is not affected by the cost of recomputing answers for usurped subgoals and degenerates into a mostly sequential evaluation where threads wait for the completion of SCCs.

\section{Discussion}

Local SLG $_{C}$ is well suited for multi-threaded evaluations that benefit from Local evaluation and can provide speedups on problems that can be subdivided relatively easily. At the same time, Local $\mathrm{SLG}_{C}$ is not intended to support general table parallelism. Local evaluation itself prevents one thread from consuming answers concurrently produced by another thread if the consuming and producing subgoals are in different SCCs. Beyond this, a Local SLG $_{C}$ evaluation may have a number of threads suspended on incomplete or usurped subgoals, although Theorem 6 puts a limit on the number of USURPATION operations.

We believe that a salient strength of Local $\mathrm{SLG}_{C}$ is its formal basis. By Theorem 7 several threads can cooperate to correctly compute the well-founded semantics, and by Theorem 6 the abstract complexity is the same as a sequential SLG evaluation. By Theorem 8 each thread in a Local SLG $_{C}$ evaluation will have a single independent SCC, and so each thread will have properties of a Local evaluation, including the space efficiency property of Theorem 4. By Corollary 2 (and Theorem 8) each thread will only return answers from completed tables, a useful property for computing the well-founded semantics and answer subsumption. As noted in Section 4, the implementation of Local SLG $\mathrm{S}_{C}$ directly relies on Theorem 9 and Corollary 1. As a result of the theory-oriented design, the implementation of Local $\mathrm{SLG}_{C}$, although delicate, mainly requires about 300 lines of code to be added to the tabletry instruction: thus Local SLG S $_{C}$ should be relatively easy to port to other tabling engines that implement Local evaluation.

Related Work These strengths and limitations distinguish (Local) SLG $_{C}$ from previous work, which we briefly discuss (see [6] for more details). [5] presents 
an approach to distributed tabling in which the $S D G$ (Definition 8) is distributed among threads, the dependencies partially represented by numerical encodings associated with subgoals, and a message-counting algorithm used for termination detection. Maintaining the distributed $S D G$ leads to an approach that is cubic in the number of messages. SLG ${ }_{C}$ differs from [5] in being a more minimal extension of SLG requiring only the addition of markings and USURPATION, and in retaining the complexity of SLG. Another distributed tabling method, [2] avoids the cubic message complexity by using a centralized table manager to maintain dependency and other information and a credit-recovery algorithm to detect completion of the SCCs. SLG ${ }_{C}$ differs from [2] in not requiring an explicit table manager and in using the "optimistic" UsURPATION operation to control concurrency, as well as in being a formalism sufficient for proving completeness and other properties. [9] presents algorithms for adding tabling to an or-parallel engine and implements these algorithms in YAP, with impressive results for definite programs. As mentioned above, unlike [9] Local SLG $_{C}$ does not address general table parallelism, although it addresses normal programs and is based on a formalization which permits a concise implementation. Perhaps the closest work is [3] which allows threads to share answers when tables are not completed: Concurrent SLG differs from this work in using a simpler method of concurrency control, as well as in modeling normal rather than definite programs.

Acknowledgements: The authors thank Manuel Carro, Pablo Chico de Guzmán, and anonymous reviewers for their careful comments.

\section{References}

1. W. Chen and D. S. Warren. Tabled Evaluation with Delaying for General Logic Programs. Journal of the ACM, 43(1):20-74, January 1996.

2. C. Damásio. A distributed tabling system. In Proceedings of the 2nd Workshop on Tabulation in Parsing and Deduction, TAPD'2000, pages 65-75, 2000.

3. J. Freire, R. Hu, T. Swift, and D. S. Warren. Parallelizing tabled evaluation. In 7th International PLILP Symposium, pages 115-132. Springer-Verlag, 1995.

4. J. Freire, T. Swift, and D. S. Warren. Beyond depth-first: Improving tabled logic programs through alternative scheduling strategies. JFLP, 1998(3), 1998.

5. R. Hu. Distributed Tabled Evaluation. PhD thesis, SUNY at Stony Brook, 1997.

6. R. Marques. Concurrent Tabling: Algorithms and Implementation. PhD thesis, Universidade Nova de Lisboa, 2007.

7. R. Marques, T. Swift, and J. Cunha. Extending tabled logic programming with multi-threading: A systems perspective. 2008.

8. R. Marques, T. Swift, and J. Cunha. A simple and efficient implementation of concurrent local tabling. Available at http://www.cs. sunysb.edu/ ${ }^{\sim}$ swift, 2008.

9. R. Rocha, F. Silva, and V. S. Costa. On applying or-parallelism and tabling to logic programs. Theory and Practice of Logic Programming, 4(6), 2004.

10. R. Rocha, F. Silva, and V. Santos Costa. Dynamic mixed-strategy evaluation of tabled logic programs. In ICLP, page 250264, 2005.

11. J. Spencer. The Strange Logic of Random Graphs. Springer, 2000.

12. A. van Gelder, K.A. Ross, and J.S. Schlipf. Unfounded sets and well-founded semantics for general logic programs. Journal of the ACM, 38(3):620-650, 1991. 